\title{
Probing new electroweak states via precision measurements at the LHC and future colliders
}

\author{
Luca Di Luzio, ${ }^{a, b}$ Ramona Gröber ${ }^{c, b}$ and Giuliano Panico ${ }^{d, e}$ \\ ${ }^{a}$ Dipartimento di Fisica, Università di Pisa and INFN, \\ Pisa, Italy \\ ${ }^{b}$ Institute for Particle Physics Phenomenology, Department of Physics, Durham University, \\ DH1 3LE, Durham, United Kingdom \\ ${ }^{c}$ Humboldt-Universität zu Berlin, Institut für Physik, \\ Newtonstr. 15, 12489 Berlin, Germany \\ ${ }^{d}$ Deutsches Elektronen-Synchrotron (DESY), \\ Notkestr. 85, 22607 Hamburg, Germany \\ e IFAE and BIST, Universitat Autónoma de Barcelona, \\ E-08193 Bellaterra, Barcelona, Spain \\ E-mail: lukaluz@gmail.com, ramona.groeber@physik.hu-berlin.de, \\ gpanico@ifae.es
}

ABSTRACT: Several new physics scenarios, motivated e.g. by dark matter, feature new electroweakly charged states where the lightest particle in the multiplet is stable and neutral. In such cases direct searches at LHC are notoriously difficult, while electroweak precision tests both at hadron and lepton colliders offer the possibility to indirectly probe those states. In this work, we assess the sensitivity of the high-luminosity phase of the LHC on new electroweak multiplets via the modification of neutral and charged Drell-Yan processes, and compare the reach of future hadron and lepton colliders presently under consideration.

KEYwords: Beyond Standard Model, Supersymmetric Standard Model

ArXiv EPRINT: 1810.10993 


\section{Contents}

1 Introduction 1

2 Physics case for new EW multiplets 3

2.1 Minimal (milli-charged) Dark Matter 3

2.2 Accidental matter 5

3 Universal EW corrections to $2 \rightarrow 2$ fermion processes 5

3.1 Form factors 6

$\begin{array}{lll}3.2 & \text { Modification of the SM amplitude } & 7\end{array}$

4 Prospects at the HL-LHC and future hadron colliders $\quad 8$

4.1 Description of the analysis 8

4.2 Results: HL-LHC 8

$\begin{array}{lll}\text { 4.2.1 LO vs. NNLO } & 10\end{array}$

$\begin{array}{ll}\text { 4.2.2 Impact of systematic uncertainties } & 10\end{array}$

$\begin{array}{lll}4.3 & \text { Results: future hadron colliders } & 11\end{array}$

5 Prospects at future lepton colliders $\quad 12$

$\begin{array}{lll}5.1 & \text { Results: } e^{+} e^{-} \text {collider } & 14\end{array}$

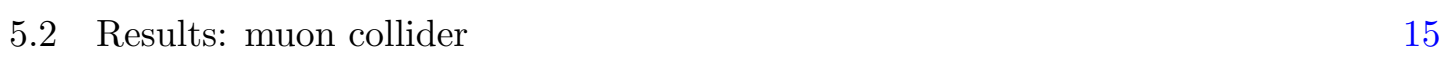

6 Comparison with direct searches $\quad 15$

$\begin{array}{llr}7 & \text { Conclusions } & 18\end{array}$

$\begin{array}{lr}\text { A Perturbativity } & 19\end{array}$

B Additional results for future hadron colliders 20

\section{Introduction}

Massive particles carrying electroweak (EW) quantum numbers are predicted in many motivated extensions of the standard model (SM). For instance, neutralinos and charginos in supersymmetry (SUSY) or vector-like leptons in composite Higgs models. Being only charged under $\mathrm{SU}(2)_{L} \times \mathrm{U}(1)_{y}$, they remain more elusive at the LHC with respect to coloured states, with current bounds well below the TeV scale. EW multiplets are particularly difficult to be probed in collider experiments whenever the lightest particle of the $\mathrm{SU}(2)_{L} n$-plet is electrically neutral and stable. The current bounds, stemming from disappearing track and mono-X searches are of the order of $100-500 \mathrm{GeV}$ (depending on the 
dimensionality $n$ ). On the other hand, this latter case is also of particular interest since it renders the lightest particle in the $n$-plet an ideal candidate for dark matter (DM). To this end, alternative ways of probing EW multiplets are particularly welcome.

In this paper we explore the possibility of indirectly probing new EW states through the precise measurements of neutral and charged Drell-Yan processes: $p p \rightarrow \ell^{+} \ell^{-}$and $p p \rightarrow \ell \nu$. In ref. [1] it was shown that the latter processes can be exploited at the LHC in order to perform accurate tests of the EW sector, by benefitting from the growth in energy of the corrections due to new physics (see also [2,3]). In ref. [1] the limit in which new physics is significantly heavier than the available collider energy was considered. In this case, if new physics affects mainly the gauge boson self energies, the corrections can be encoded in the "oblique" parameters, namely the original Peskin-Takeuchi $S, T$ and $U$ [4], extended by the additional parameters $W$ and $Y$ [5] which include higher-order terms in the momentum expansion (for an updated EW fit employing low-energy observables see e.g. ref. [6]). On the other hand, the $W$ and $Y$ parameters induce corrections that grow in energy, therefore they can be easily accessed at the LHC by exploiting the high reach in invariant mass $m_{\ell \ell}$ or transverse mass $m_{T}$. This allows to significantly improve the sensitivity with respect to LEP. On the contrary $S, T$ and $U$ give an overall rescaling of the cross section, thus they are dominantly tested at the $Z$ pole. In this case LHC can hardly compete with LEP. ${ }^{1}$

When the new physics is very light, much below the effective collider energy, the simplified description in terms of oblique parameters is not appropriate anymore. In this case the effects of light new physics can be seen as a modification of the running of the EW gauge couplings due to the change in the SM beta functions for scales $\mu>m$, where $m$ generically denotes the mass scale of the new state. The regime of very light new physics has been analysed e.g. in refs. [11-13].

Since the current bounds on EW multiplets are of the order of few $100 \mathrm{GeV}$ we are mostly interested in an intermediate regime where none of the two limits above can be applied. We will instead keep the full corrections due to the new state in the gauge boson propagators. Such an analysis has been done previously in ref. [14] in the context of future $e^{+} e^{-}$lepton colliders and in ref. [15] for LHC and its high-luminosity phase (HL-LHC). While ref. [15] considers only the neutral Drell-Yan production, we include in our analysis also the charged Drell-Yan process and show that it actually leads to stronger bounds than the neutral channel. We pay special attention to the treatment of uncertainties and show where an improvement of systematic errors and/or parton distribution functions (PDF) can lead to a substantial improvement of the bound. Furthermore, we analyse the sensitivity of future facilities presently under discussion, such as a $28 \mathrm{TeV}$ high-energy LHC (HELHC) [16] and a $100 \mathrm{TeV}$ future circular collider (FCC-100) [17, 18], as well as high-energy lepton colliders including $e^{+} e^{-}$machines like the Compact Linear Collider (CLIC) [19] and muon colliders, with the multi-TeV options MAP (muon accelerator program) [20] based

\footnotetext{
${ }^{1}$ In refs. [7-9] it was shown that also precision measurements of di-boson production can provide further tests of the SM effective theory. Exploitation of these channels allows LHC to reach a sensitivity comparable to the LEP one also for observables analogous to the $S$ parameter. Further indirect tests of dark fermions or scalars can come from Higgs coupling measurements [10].
} 
on proton scattering on a target and LEMMA (low emittance muon accelerator) [21-23] based on positron scattering on a target.

The paper is structured as follows. In section 2 we introduce the physics case for EW multiplets. In section 3 we describe the parametrization of the new physics corrections to the gauge boson propagators. In section 4 we present the analysis for the HL-LHC and future hadron colliders which represents the central part of our work, while in section 5 we study the sensitivity of future lepton collider options. Finally in section 6 we briefly compare our bounds on EW multiplets with direct searches and conclude in section 7. Appendices $\mathrm{A}$ and $\mathrm{B}$ are devoted to the collection of some additional results and technical details.

\section{Physics case for new EW multiplets}

New EW states charged under $\mathrm{SU}(2)_{L} \times \mathrm{U}(1)_{y}$, which are generically denoted by their quantum numbers $\chi \sim(1, n, y)$, with the three entries denoting the $\mathrm{SU}(3)_{c} \times \mathrm{SU}(2)_{L} \times \mathrm{U}(1)_{y}$ representation, appear in many motivated beyond-the-SM scenarios. The EW sector of SUSY comprising the wino/higgsino system is certainly one of the most compelling cases. Larger multiplets with $n>3$ can also be motivated by DM, if the lightest particle in the $n$-dimensional multiplet is stable and neutral. In the following, we briefly review a few frameworks which motivate the existence of large EW multiplets from the standpoint of accidental global symmetries.

\subsection{Minimal (milli-charged) Dark Matter}

The idea behind Minimal Dark Matter (MDM) [24-26] is to introduce a single EW multiplet $\chi$ which is accidentally stable at the renormalizable level due to the SM gauge symmetry. One further assumes $y=0$ (to avoid direct detection bounds from $Z$ exchange) and that the lightest particle in the multiplet is neutral. The latter is actually an automatic feature if the mass splitting within the $n$-plet is purely radiative as in the case of fermions with $n>3$. On the contrary, scalars can receive a model-dependent tree-level splitting from the scalar potential, which we assume to be subleading. The contribution to the relic density is completely fixed by the EW gauge interactions and the mass of the new state $m_{\chi}$, thus making the framework extremely predictive.

If one further requires that the theory remains weakly coupled up to the Planck scale and that the gauge quantum numbers of $\chi$ are such that no operators with dimension smaller than 6 can mediate the decay of $\chi{ }^{2}$ only one multiplet is allowed, namely the Majorana fermion representation $(1,5,0)_{\mathrm{MF}} \cdot{ }^{3}$ To be completely general, in the following, we will however consider multiplets of different kind, namely in the real scalar, complex scalar, Majorana fermion, Dirac fermion representations, which we denote by the labels RS, CS, MF, DF respectively.

\footnotetext{
${ }^{2}$ Operators with dimension $\leq 5$ would lead to a too fast $\chi$ decay, even with a Planck scale cutoff.

${ }^{3}$ Originally also the real scalar representation $(1,7,0)_{\mathrm{RS}}$ was included in the list, but it was shown later in ref. [27] that a previously overlooked $d=5$ operator leads to a loop-induced decay of $\chi$, with a lifetime shorter than the age of the Universe.
} 


\begin{tabular}{|l|c|c|c|c|c|c|}
\hline$\chi / m_{\chi}[\mathrm{TeV}]$ & $\mathrm{DM}$ & HL-LHC & HE-LHC & FCC-100 & CLIC-3 & Muon-14 \\
\hline$(1,2,1 / 2)_{\mathrm{DF}}$ & 1.1 & - & - & - & 0.4 & 0.6 \\
$(1,3, \epsilon)_{\mathrm{CS}}$ & 1.6 & - & - & - & 0.2 & 0.2 \\
$(1,3, \epsilon)_{\mathrm{DF}}$ & 2.0 & - & 0.6 & 1.5 & $0.8 \&[1.0,2.0]$ & $2.2 \&[6.3,7.1]$ \\
$(1,3,0)_{\mathrm{MF}}$ & 2.8 & - & - & 0.4 & $0.6 \&[1.2,1.6]$ & 1.0 \\
$(1,5, \epsilon)_{\mathrm{CS}}$ & 6.6 & 0.2 & 0.4 & 1.0 & $0.5 \&[0.7,1.6]$ & 1.6 \\
$(1,5, \epsilon)_{\mathrm{DF}}$ & 6.6 & 1.5 & 2.8 & 7.1 & 3.9 & 11 \\
$(1,5,0)_{\mathrm{MF}}$ & 14 & 0.9 & 1.8 & 4.4 & 2.9 & $3.5 \&[5.1,8.7]$ \\
$(1,7, \epsilon)_{\mathrm{CS}}$ & 16 & 0.6 & 1.3 & 3.2 & 2.4 & $2.5 \&[3.5,7.4]$ \\
$(1,7, \epsilon)_{\mathrm{DF}}$ & 16 & 2.1 & 4.0 & 11 & 6.4 & 18 \\
\hline
\end{tabular}

Table 1. Pure higgsino/wino-like DM and MDM candidates, together with the corresponding masses saturating the DM relic density (second column) and the projected 95\% CL exclusion limits from EW precision tests at HL-LHC, HE-LHC, FCC-100, CLIC-3 and Muon-14 (see text for details about center-of-mass energies and luminosities). In the last two columns the numbers in square brackets stand for a mass interval exclusion. The cases where the DM hypothesis could be fully tested are emphasized in light red.

The MDM framework was extended in ref. [28] to contemplate the possibility of a milli-charge $\epsilon \ll 1$. Bounds from DM direct detection imply $\epsilon \lesssim 10^{-9}$. The milli-charge has hence no bearings for collider phenomenology, but it ensures the (exact) stability of the lightest particle in the EW multiplet due to the SM gauge symmetry, in the same spirit of the original MDM formulation. A notable feature of the milli-charged scenario is that the contribution of the complex multiplet to the relic density gets doubled compared to the case of a single real component (thus making the thermal mass roughly a factor $\sqrt{2}$ smaller). On the other hand, the number of degrees of freedom are also doubled, thus improving the indirect testability of those scenarios via EW precision tests at colliders.

The MDM candidates (including for completeness also the higgsino-like $(1,2,1 / 2)_{\mathrm{DF}}$ and wino-like $(1,3,0)_{\mathrm{MF}} \mathrm{DM}$, which require a stabilization mechanism beyond the SM gauge symmetry) are summarized in table 1, together with their thermal mass saturating the DM relic density ${ }^{4}$ and the projected $95 \%$ confidence level (CL) exclusion limits of five representative future colliders: HL-LHC $(\sqrt{s}=14 \mathrm{TeV}$ and $L=3 / \mathrm{ab})$, HE-LHC $(\sqrt{s}=$ $28 \mathrm{TeV}$ and $L=10 / \mathrm{ab})$, FCC-100 $(\sqrt{s}=100 \mathrm{TeV}$ and $L=20 / \mathrm{ab})$, CLIC-3 $(\sqrt{s}=3 \mathrm{TeV}$ and $L=4 / \mathrm{ab})$, Muon-14 $(\sqrt{s}=14 \mathrm{TeV}$ and $L=20 / \mathrm{ab})$. The details of the analysis will be presented in sections $4-5$.

We can anticipate here some results of our analysis. The HL-LHC and the HE-LHC are not able to test any of the DM candidates for masses which allow these multiplets to saturate the whole DM relic density. The FCC-100, on the other hand, could fully test

\footnotetext{
${ }^{4}$ The thermal masses in the $\epsilon=0$ cases are extracted from ref. [29] which takes into account both Sommerfeld enhancement and bound state formation effects. In the cases $\epsilon \neq 0$ we quote instead the results from ref. [28], which however do not include effects from bound state formation that are expected to sizeable for $n \gtrsim 5$ (e.g. in the case of $(1,5,0)_{\mathrm{MF}}$ the inclusion of bound state effects leads to a $20 \%$ increase of the thermal mass [29]).
} 
the $(1,5, \epsilon)_{\mathrm{DF}}$ candidate and would come close to test the interesting mass range for the $(1,3, \epsilon)_{\mathrm{DF}}$ and $(1,7, \epsilon)_{\mathrm{DF}}$ multiplets. Lepton colliders are usually better at testing small multiplets, which are difficult to probe at hadron colliders. CLIC-3 and Muon-14 could fully test the $(1,3, \epsilon)_{\mathrm{DF}}$ multiplet. Muon-14 would also surpass the FCC-100 sensitivity on both the $(1,5, \epsilon)_{\mathrm{DF}}$ and the $(1,7, \epsilon)_{\mathrm{DF}}$ multiplets, reaching the masses that saturate the DM relic density. It could also test a significant fraction of the mass range for the $(1,5,0)_{\mathrm{MF}}$ multiplet.

\section{$2.2 \quad$ Accidental matter}

From a more phenomenological point of view, larger multiplets with $n>3$ are also motivated by the fact that they automatically respect the accidental and approximate symmetry structure of the SM and are hence screened from low-energy probes such as baryon and lepton number and flavour/CP violating processes. Particularly interesting multiplets are the ones that satisfy the following requirements [27]: i) automatically preserve the accidental and approximate symmetry structure of the $\mathrm{SM} ; \mathrm{ii}$ ) are cosmologically viable; $i i i$ ) form consistent effective field theories (EFTs) with a cut-off scale as high as $10^{15} \mathrm{GeV}$ (as suggested by neutrino masses). These multiplets are easily compatible with the stringent flavor tests and typically present peculiar collider signatures, for instance long-lived particles. A finite list of multiplets satisfy the above criteria [27], and among those a subset feature a neutral lightest state: $(1,5,0)_{\mathrm{RS}},(1,5,1)_{\mathrm{CS}},(1,5,2)_{\mathrm{CS}},(1,7,0)_{\mathrm{RS}},(1,4,3 / 2)_{\mathrm{DF}}$, $(1,5,0)_{\mathrm{MF}}$. These multiplets are hence a natural target for our study. It turns out that the value of the hypercharge, unless exotically large, plays a subleading role for the extraction of the bound. ${ }^{5}$ Hence, instead of reporting explicitly the projected reach for all the accidental matter candidates, we refer directly to the results in sections $4-5$.

\section{Universal EW corrections to $2 \rightarrow 2$ fermion processes}

In this work we consider the universal EW corrections to $2 \rightarrow 2$ processes involving SM fermions in the final state, stemming from a new scalar/fermion multiplet $\chi \sim(1, n, y)$. We further assume that $i$ ) $\chi$ does not interact at the renormalizable level with the SM matter fields and $i i$ ) the mass splitting within the $n$-plet is negligible. While the former assumption ensures that the radiative corrections can be encoded into the universal modification of the EW gauge boson propagators, the latter one is a simplification which becomes asymptotically good in the regime $m_{\chi} \gg m_{Z}$, relevant for future colliders. It is important to stress, however, that the above assumptions are automatically satisfied for fermions with $n>3$, while in the case of scalars they further require that interaction terms with the SM Higgs are subleading.

The most useful observables at hadron colliders turn out to be the neutral and charged DY processes with leptons $\ell=e, \mu$ in the final states: $p p \rightarrow \ell^{+} \ell^{-}$and $p p \rightarrow \ell \nu$, while at lepton colliders one can consider the neutral current processes $\ell^{+} \ell^{-} \rightarrow f \bar{f}$ (with $f$ denoting a SM fermion). In the following, we describe the formalism for deriving the modified EW

\footnotetext{
${ }^{5}$ E.g. in the case of $(1,5,2)_{\mathrm{CS}}$ the bound on the mass gets strengthen by $5 \%$ compared to $(1,5,0)_{\mathrm{CS}}$.
} 
gauge boson propagators, which is common to all $2 \rightarrow 2$ fermion processes. For related analyses see also refs. $[14,15]$.

\subsection{Form factors}

The modifications of the EW gauge boson propagators due to the new state $\chi \sim(1, n, y)$ is parametrized via the inclusion of the following form factors in the effective Lagrangian

$$
\mathcal{L}_{\text {eff }}=\mathcal{L}_{\mathrm{SM}}+\frac{g^{2} C_{W W}^{\mathrm{eff}}}{8} W_{\mu \nu}^{a} \Pi\left(-D^{2} / m_{\chi}^{2}\right) W^{a \mu \nu}+\frac{g^{\prime 2} C_{B B}^{\mathrm{eff}}}{8} B_{\mu \nu} \Pi\left(-\partial^{2} / m_{\chi}^{2}\right) B^{\mu \nu},
$$

where

$$
C_{W W}^{\mathrm{eff}}=\kappa\left(n^{3}-n\right) / 6, \quad C_{B B}^{\mathrm{eff}}=2 \kappa n y^{2},
$$

and $\kappa=1 / 2,1,4,8$, respectively for $\chi$ being a real scalar (RS), complex scalar (CS), Majorana fermion (MF), Dirac fermion (DF).

The contribution of $\chi$ to the EW gauge boson propagators is purely transversal and the $\overline{\mathrm{MS}}$ renormalized form factors are (respectively for the case of a scalar and a fermion running in the loop):

$$
\begin{aligned}
& \Pi_{S}(x)=-\frac{3 x \log \left(\frac{\mu^{2}}{m_{\chi}^{2}}\right)+8(x-3)+3 x\left(\frac{x-4}{x}\right)^{3 / 2} \log \left(\frac{1}{2}\left(\left(\sqrt{\frac{x-4}{x}}-1\right) x+2\right)\right)}{144 \pi^{2} x}, \\
& \Pi_{F}(x)=-\frac{3 x \log \left(\frac{\mu^{2}}{m_{\chi}^{2}}\right)+12+5 x+3 \sqrt{\frac{x-4}{x}}(x+2) \log \left(\frac{1}{2}\left(\left(\sqrt{\frac{x-4}{x}}-1\right) x+2\right)\right)}{288 \pi^{2} x} .
\end{aligned}
$$

Here $x=q^{2} / m_{\chi}^{2}$, where $q$ is the external momentum of the gauge boson propagator and $\mu$ is the renormalization scale. A useful choice is $\mu=m_{\chi}$, which ensures that the form factors $\Pi_{S, F}$ vanish for $x=0$. This choice is henceforth assumed. The behavior of the $\Pi_{S, F}$ form factors is shown in figure 1.

In the EFT limit, $x \ll 1$, the expanded form factor is $\Pi(x) \simeq-x /\left(480 \pi^{2}\right)$, both for scalar and fermions. Since $\Pi(0)=0$ there is no contribution to the oblique parameters $S$, $T, U$ [4], while $W$ and $Y$ [5], which correspond to the Wilson coefficients of the dimension-6 operators $-\frac{W}{4 m_{W}^{2}}\left(D_{\rho} W_{\mu \nu}^{a}\right)^{2}$ and $-\frac{Y}{4 m_{W}^{2}}\left(\partial_{\rho} B_{\mu \nu}\right)^{2}$, are given by

$$
W=\frac{g^{2} C_{W W}^{\mathrm{eff}}}{960 \pi^{2}} \frac{m_{W}^{2}}{m_{\chi}^{2}}, \quad Y=\frac{g^{2} C_{B B}^{\mathrm{eff}}}{960 \pi^{2}} \frac{m_{W}^{2}}{m_{\chi}^{2}} .
$$

For $x \gtrsim 1$ the EFT breaks down and hence the full momentum dependence of the form factor must be taken into account. For $x \geq 4$ the momentum is above the pair-production threshold and the form factors develop an imaginary part (cf. figure 1).

It is interesting to notice that for $1 \lesssim x \lesssim 4$ the full form factors are (significantly) larger than the EFT approximation (compare the blue and red lines with the black line in figure 1). This means that indirect searches for multiplets with a mass close to the pair production threshold tend to be significantly more sensitive than what the EFT approximation would suggest. 


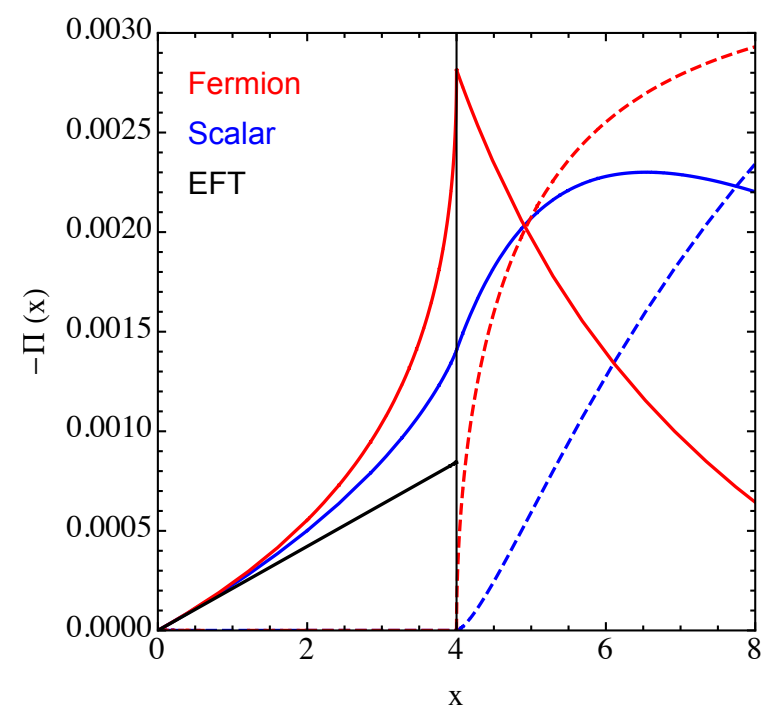

Figure 1. Kinematical dependence of the form factor for fermions (red) and scalars (blue) running in the loop, and in the EFT limit (black). Full and dashed lines denote respectively real and imaginary part of the form factor.

\subsection{Modification of the SM amplitude}

In order to derive the radiative corrections to the neutral and charged current $2 \rightarrow 2$ fermion processes, we project eq. (3.1) onto the gauge boson mass eigenstates $\gamma, Z, W$

$$
\mathcal{L}_{\text {eff }}=\mathcal{L}_{\mathrm{SM}}+\sum_{V, V^{\prime}=\gamma, Z} \frac{d_{V V^{\prime}}}{4} V_{\mu \nu} \Pi\left(-\partial^{2} / m_{\chi}^{2}\right) V^{\prime \mu \nu}+\frac{d_{W W}}{2} W_{\mu \nu}^{+} \Pi\left(-\partial^{2} / m_{\chi}^{2}\right) W^{-\mu \nu},
$$

where $d_{\gamma \gamma}=\left(e^{2} / 2\right)\left(C_{W W}^{\mathrm{eff}}+C_{B B}^{\mathrm{eff}}\right), d_{Z Z}=\left(g_{Z}^{2} / 2\right)\left(\cos ^{4} \theta_{W} C_{W W}^{\mathrm{eff}}+\sin ^{4} \theta_{W} C_{B B}^{\mathrm{eff}}\right), d_{\gamma Z}=d_{Z \gamma}=$ $\left(e g_{Z} / 2\right)\left(\cos ^{2} \theta_{W} C_{W W}^{\text {eff }}-\sin ^{2} \theta_{W} C_{B B}^{\mathrm{eff}}\right), d_{W W}=\left(g^{2} / 2\right) C_{W W}^{\mathrm{eff}}$, and we used the definitions $e=$ $g \sin \theta_{W}, g_{Z}=g / \cos \theta_{W}$ and $\tan \theta_{W}=g^{\prime} / g$. The modified EW gauge boson propagators for neutral and charged currents read respectively (keeping only the transverse part which is affected by new physics)

$$
\begin{aligned}
& \Delta_{\mu \nu}^{V V^{\prime}}=i\left[\begin{array}{cc}
\left(1-d_{\gamma \gamma} \Pi(x)\right) q^{2} & -d_{\gamma Z} \Pi(x) q^{2} \\
-d_{Z \gamma} \Pi(x) q^{2} & \left(1-d_{Z Z} \Pi(x)\right) q^{2}-m_{Z}^{2}
\end{array}\right]^{-1}\left(-g_{\mu \nu}+\frac{q_{\mu} q_{\nu}}{q^{2}}\right), \\
& \Delta_{\mu \nu}^{W W}=\frac{i}{q^{2}-m_{W}^{2}-d_{W W} q^{2} \Pi(x)}\left(-g_{\mu \nu}+\frac{q_{\mu} q_{\nu}}{q^{2}}\right) .
\end{aligned}
$$

Given the SM amplitude for a $2 \rightarrow 2$ fermion process, the effects of the new particle $\chi$ can be systematically accounted for by substituting the tree-level EW gauge boson propagators with the modified ones in eqs. (3.7)-(3.8). The leading correction to the SM cross-section comes from the interference with the SM amplitude, therefore it is due to real part of the form factor.

Note, also, that the contribution of the coefficient $C_{W W}^{\text {eff }}$ typically gives the strongest constraint (this is for instance the case for the EW states introduced in section 2). The 
reason depends in part on the hierarchy of the gauge couplings $g^{2} / g^{\prime 2} \sim 3$ and, more in general, on the fact that in the large $n$ and $y$ limit the effective coefficients scale like $C_{W W}^{\mathrm{eff}} \sim n^{3}$ and $C_{B B}^{\mathrm{eff}} \sim n y^{2}$. So, unless the hypercharge is exotically large (or both $n$ and $y$ are exotically large) the contribution of $C_{B B}^{\mathrm{eff}}$ is subleading. Moreover, one expects that for some large values of $n$ or $y$ perturbativity breaks down. This issue is analysed in appendix A, where we find that $n$ up to 9 can still be considered to be in the perturbative domain.

Although we will present the mass exclusions as a function of the dimensionality $n$ of an irreducible $\mathrm{SU}(2)_{L}$ representation, it is possible to recast our results for a generic $\mathrm{SU}(2)_{L}$ reducible representation by properly rescaling the coefficient $C_{W W}^{\mathrm{eff}}$ (cf. eq. (3.2)). E.g. $N$ copies of fundamentals $(n=2)$ with degenerate mass would effectively correspond to a single representation with $n_{\star}$ obtained by solving $n_{\star}^{3}-n_{\star}=N\left(2^{3}-2\right)$.

\section{Prospects at the HL-LHC and future hadron colliders}

In this section we report the numerical results of our analysis in the context of hadron colliders. First of all we focus on the HL-LHC, then we consider possible future highenergy hadron colliders, in particular the HE-LHC and FCC-100.

\subsection{Description of the analysis}

We perform a simple analysis based on a cut-and-count strategy. In the case of the neutral $\ell^{+} \ell^{-}$process we exploit the distribution in the invariant mass of the lepton pair, whereas in the case of the charged process $\ell \nu$ we consider the distribution in the transverse mass of the event (one could equivalently use the $p_{T}$ distribution of the charged lepton).

For simplicity we do not take into account the angular and rapidity distributions, which could be accessed in the $\ell^{+} \ell^{-}$process. We expect this information not to play a relevant role in our analysis. In fact, since the leading effects of EW multiplets at hadron colliders are driven by contributions to $C_{W W}^{\mathrm{eff}}$, the angular and rapidity distributions are only marginally distorted. The contributions coming from $C_{B B}^{\text {eff }}$, which could be more easily distinguished through an angular analysis, are instead very suppressed and can typically be neglected.

For both invariant mass and transverse mass distributions we use a binned loglikelihood analysis. The size of the bins is chosen to be $15 \%$ of their lower boundary and we only considered events above the $200 \mathrm{GeV}$ threshold in order to avoid large effects from real $Z$ or $W$ production. The boundaries of the bins are thus given by $(200 \mathrm{GeV}) \times 1.15^{n}$. For the HL-LHC we include in the analysis bins up to $\sim 2 \mathrm{TeV}$, while for the HE-LHC and FCC-hh we stop at $\sim 3.5 \mathrm{TeV}$ and $\sim 20 \mathrm{TeV}$ respectively.

The binned cross section at LO and NNLO QCD [30-34] has been evaluated through the code FEWZ 2.0 [35], using the NNPDF30 PDFs [36] with $\alpha_{s}\left(M_{Z}\right)=0.118$.

\subsection{Results: HL-LHC}

We now summarize the results we obtained for the HL-LHC. In order to properly assess the sensitivity of our analysis it is important to make realistic assumptions about the 

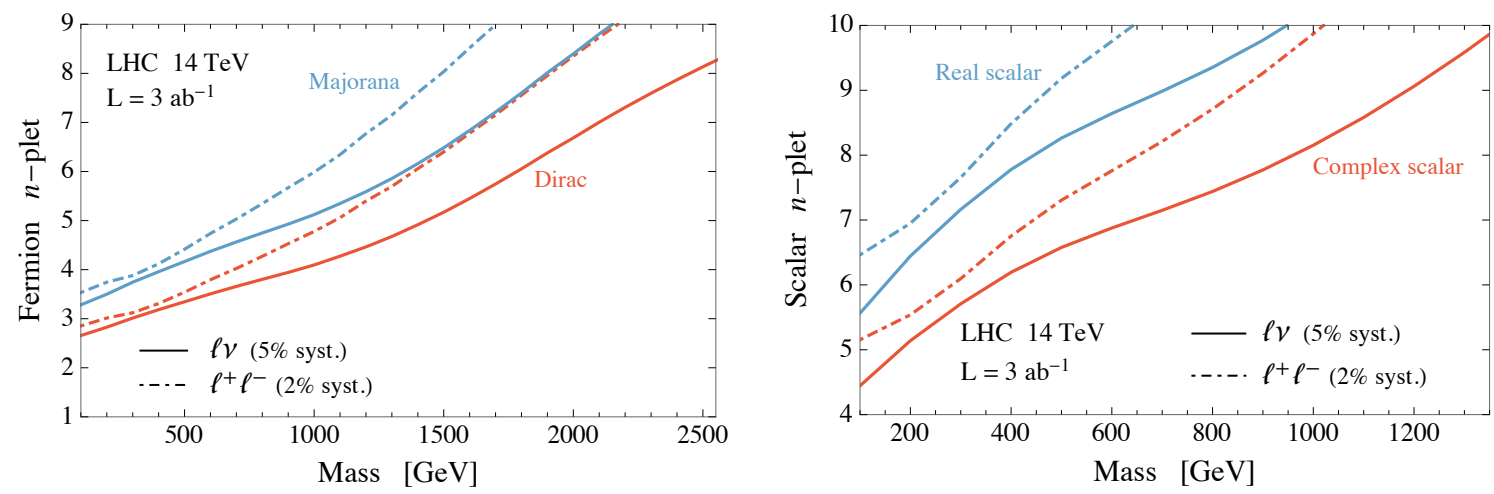

Figure 2. Expected 95\% CL exclusion limits at the HL-LHC. The left and right panels show the bounds on fermion and scalar multiplets respectively. The vertical axis reports the effective $n$ of the multiplet, while the horizontal axis gives the mass of the states in the multiplet, which are assumed to be (almost) degenerate. The solid and dot-dashed lines correspond to the bounds from the $\ell \nu$ and $\ell^{+} \ell^{-}$channels respectively. The blue (red) lines give the bounds for Majorana (Dirac) fermions on the left panel and for real (complex) scalars in the right panel.

theoretical and experimental systematic uncertainties. As benchmark targets we include in our analysis an uncorrelated and a fully correlated systematical error both at the level of $2 \%$ in the $\ell^{+} \ell^{-}$channel and $5 \%$ in the $\ell \nu$ channel. These assumptions were found to be realistic in ref. [1]. We also separately include the uncertainty in the PDFs. For simplicity we sum in quadrature the various errors.

The projection for the $95 \%$ CL exclusion bounds for fermion and scalar multiplets are shown in figure 2. The plots show the exclusions on the "effective size" of the multiplet as a function of the mass of the multiplet. For all multiplets we are considering, the main corrections to the SM cross sections come from deformations of the $\mathrm{SU}(2)_{L}$ gauge propagator (namely contributions to the $C_{W W}^{\mathrm{eff}}$ coefficient), while the effects of the hypercharge coupling are practically negligible. The effective size is thus defined by converting the bound on $C_{W W}^{\text {eff }}$ into a bound on $n$ (considered now as a real number) through eq. (3.2).

The plots report the bounds from both the $\ell \nu$ channel (solid lines) and the $\ell^{+} \ell^{-}$channel (dot-dashed lines). One can see that the charged channel gives always the best sensitivity. The difference is more noticeable for large multiplets $(n \gtrsim 4)$, while it is milder for small multiplets $(n \sim 3)$. The stronger sensitivity in the $\ell \nu$ channel is due to a combination of factors. First of all the size of the deviations in this channel are slightly larger than in the $\ell^{+} \ell^{-}$one. Moreover the cross section in the charged channel is larger, thus providing a significant improvement on the statistics, which helps especially for high masses, where the statistical uncertainty dominates over the systematic ones.

It is interesting to notice that, due to the sizable systematic uncertainty, hadron colliders cannot test small $\mathrm{SU}(2)_{L}$ multiplets even for small masses. This is due to the fact that the size of the fractional deviation with respect to the SM cross section is fully determined by the multiplet size. For small multiplets these effects are smaller than the systematic uncertainties and therefore not detectable. We will see in the following (section 4.2.2) how a change in the systematic uncertainties affects these results. 


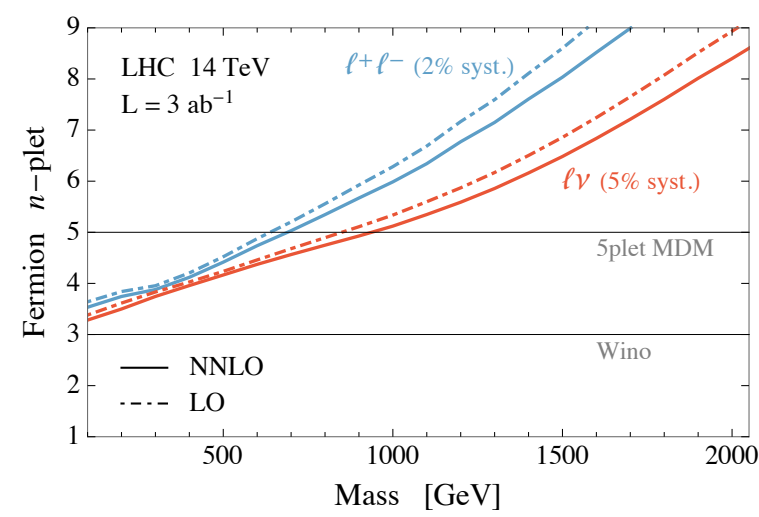

Figure 3. Comparison of the 95\% CL exclusion bounds on Majorana fermion multiplets at the HL-LHC obtained by using the LO (dot-dashed lines) and NNLO (solid lines) distributions. The blue lines refer to the $\ell^{+} \ell^{-}$process, while the red ones correspond to the $\ell \nu$ process.

The expected exclusion bounds for several multiplets which could provide a DM candidate are reported in table 1 . One can see that the HL-LHC does not allow to test any of the multiplets for mass values which saturate the DM relic abundance.

\subsubsection{LO vs. NNLO}

It is interesting to study the impact of higher-order corrections to the kinematic distributions on the exclusion bounds. For this purpose we show in figure 3 how the bounds change if we use LO or NNLO distributions to derive them.

The figure shows that the impact of higher-order corrections is relatively mild, giving an increase in the mass bound of order $5 \%-10 \%$. This is due to the fact that the higherorder corrections give a mild enhancement (of order 20\%) of the cross section. Notice that most of the enhancement is just given by the NLO QCD contributions, while the impact of the NNLO corrections on the extraction of the bounds are practically negligible. For this reason in figure 3 we did not report the NLO lines, which almost exactly overlap with the NNLO ones.

\subsubsection{Impact of systematic uncertainties}

A second aspect we want to discuss is the dependence of our results on the systematic uncertainties. Focusing again on the Majorana fermion case, we show in figure 4 how the exclusion bounds change by varying the different sources of systematic uncertainty, namely the PDF uncertainty and the additional correlated and uncorrelated errors.

One can clearly see that the impact of the PDF uncertainty is dominating for large multiplet masses, roughly above $1 \mathrm{TeV}$. In this region the PDF uncertainty becomes much larger than the other sources of systematic error. The impact of halving the PDF uncertainty provides an increase in the bounds by roughly $10 \%-15 \%$ for high masses.

On the other hand, the additional systematic uncertainties dominate for low masses. The dot-dashed yellow lines show that a factor-of-two increase of these uncertainties can significantly degrade the bounds for masses $\lesssim 1 \mathrm{TeV}$, while a reduction by a factor of two 

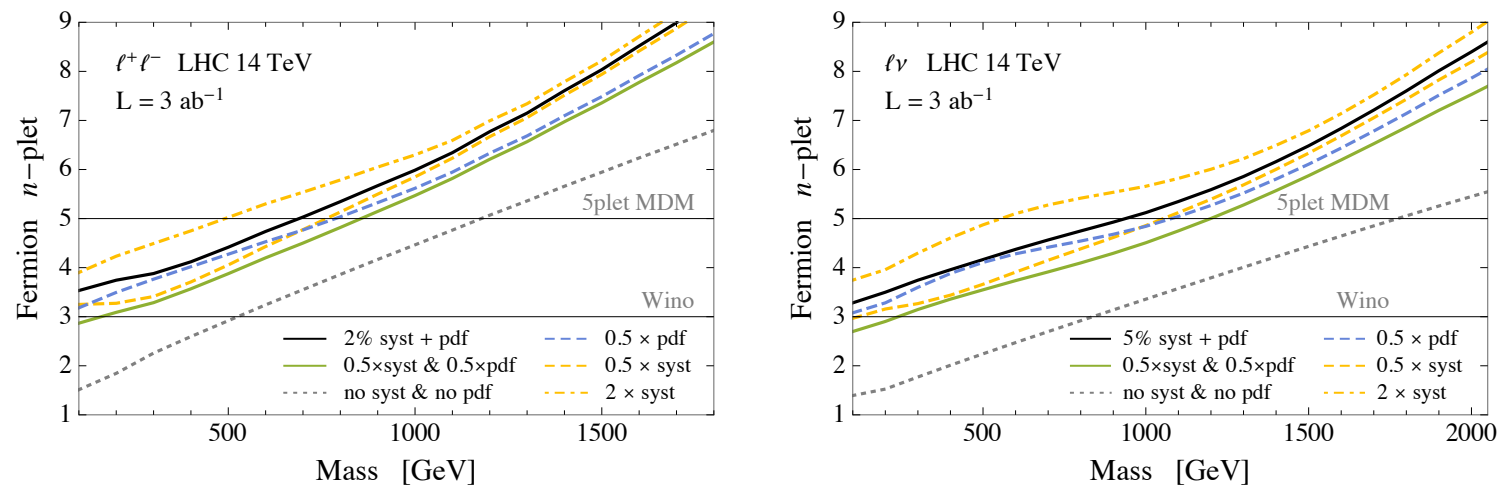

Figure 4. Dependence of the $95 \%$ CL exclusion bounds on Majorana fermion multiplets at the HL-LHC on the PDF uncertainties and on the additional systematic errors. The left and right panels show the bounds from the $\ell^{+} \ell^{-}$and $\ell \nu$ channels respectively. The solid black lines give the bounds for our benchmark systematic errors. The dashed (dot-dashed) yellow lines show how the bound changes if the additional systematic uncertainties are halved (doubled). The dashed blue lines show how the bounds are modified by halving the PDF uncertainty. The solid green lines give the bound with all systematic uncertainties halved. Finally the dotted grey lines correspond to the bounds with no systematic and PDF errors.

allows to access significantly lower $n$ for small multiplet masses or, equivalently, could allow for even a $\sim 100 \%$ improvement in the bounds at fixed $n$.

One can see that an overall reduction by $50 \%$ of all systematic uncertainties can give a significant improvement in the exclusion reach. In particular it allows us to probe the $n=3$ case, which can be interpreted as a mass-degenerate wino multiplet. This class of states is not accessible in the benchmark systematic error scenario we were considering for our main results.

Finally, mainly for illustrative purposes, we also report the bounds obtained by neglecting all systematic uncertainties (dashed grey lines). In this case, thanks to the very high statistics in the low-mass bins, even an $\mathrm{SU}(2)_{L}$ triplet $^{6}$ could be tested up to a mass $\sim 800 \mathrm{GeV}$.

\subsection{Results: future hadron colliders}

We now present the expected exclusion limits at future high-energy hadron colliders, in particular HE-LHC and FCC-100. We choose as benchmarks for the integrated luminosity $L=10 \mathrm{ab}^{-1}$ for the HE-LHC and $L=20 \mathrm{ab}^{-1}$ for FCC-100. As for the HL-LHC case, we include in the analysis the present PDF errors, as well as additional uncorrelated and fully correlated systematic uncertainties at the level of $2 \%$ for the $\ell^{+} \ell^{-}$channel and $5 \%$ for the $\ell \nu$ channel. Results for alternative luminosity benchmarks, as well as for different choices of systematic uncertainties, are reported in appendix B.

The expected bounds for the HE-LHC and FCC-100 benchmarks are shown in figure 5 and figure 6 respectively. Comparing with the HL-LHC results one can see that the HE-

\footnotetext{
${ }^{6}$ Notice that a degenerate Higgsino multiplet would correspond (neglecting the subleading effect from the hypercharge) to an effective Majorana fermion with $n=2.4$ in figure 4 .
} 

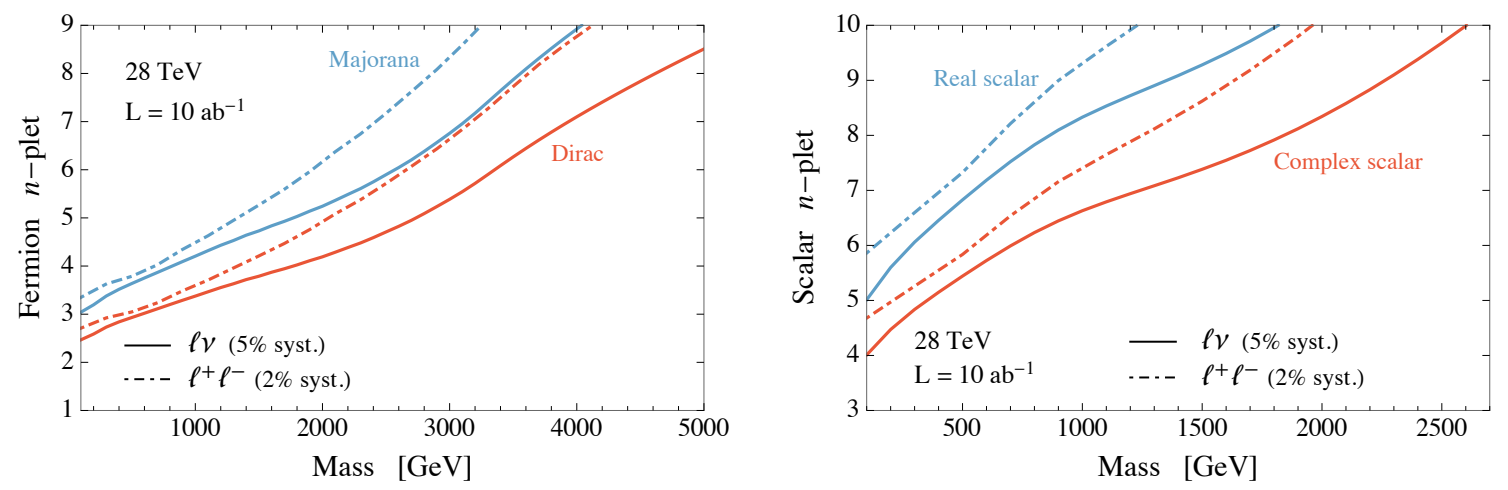

Figure 5. Expected 95\% CL exclusion limits obtained for the HE-LHC.
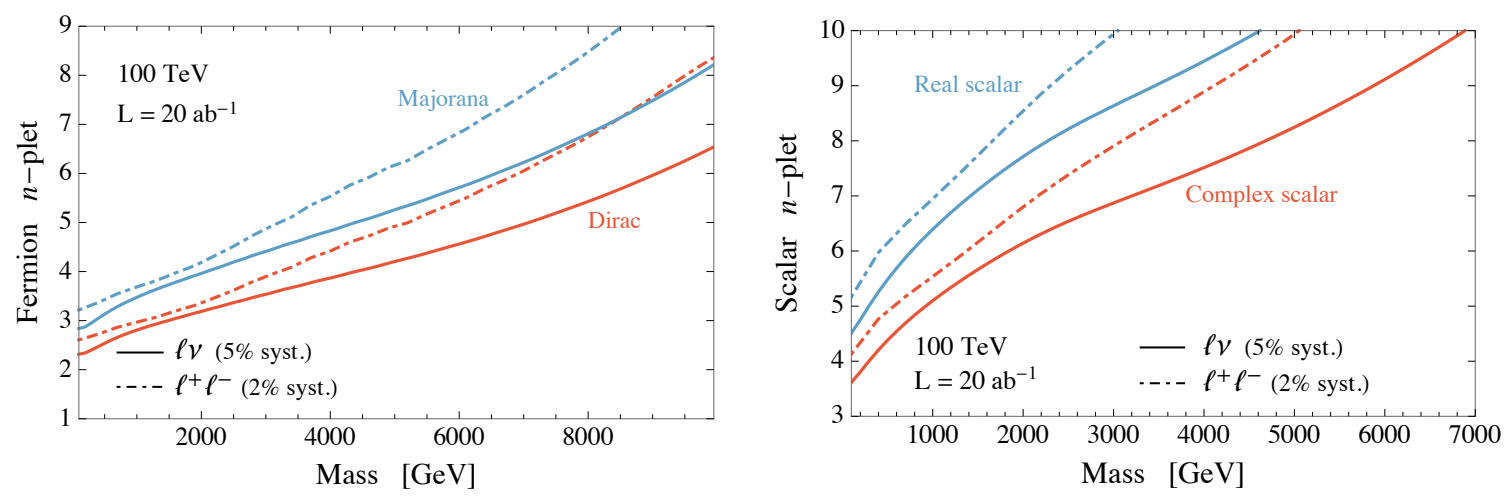

Figure 6. Expected 95\% CL exclusion limits obtained for the FCC-100.

LHC allows for an improvement of the mass bounds by roughly a factor 2, whereas FCC100 gives an improvement by roughly a factor 5 . The advantage of a larger centre-ofmass energy translates mostly in shifting the exclusion reach to higher masses, while the improvement in reaching smaller multiplet sizes is limited. EW triplets, which were out of the HL-LHC reach, can be tested at high-energy hadron colliders only for the case of Dirac fermions, whereas they remain outside the reach for the case of Majorana fermions and for multiplets of scalars.

The expected exclusion bounds for multiplets which could provide a DM candidate are reported in table 1. The HE-LHC reach is still far from probing realistic DM masses. On the other hand FCC-100 could successfully test the MDM scenario with a Dirac fermion 5plet $(1,5, \epsilon)_{\mathrm{DF}}$ and is not far from probing the triplet $(1,3, \epsilon)_{\mathrm{DF}}$ and 7-plet $(1,7, \epsilon)_{\mathrm{DF}}$ cases.

\section{$5 \quad$ Prospects at future lepton colliders}

The main difference of lepton machines compared to hadron colliders is the fact that the momentum of the EW gauge boson propagators is fixed by the centre-of-mass energy $\sqrt{s}$ (i.e. it is not smeared by the PDF). Although the most interesting region for lepton colliders is the one below the energy threshold for $\chi$ pair production, EW corrections to $2 \rightarrow 2 \mathrm{SM}$ fermion processes could also be used to probe the region above threshold, in a 
complementary way with respect to direct searches. Other advantages of $\ell^{+} \ell^{-}$machines are the reduced systematics and (in the case of electrons) the possibility of playing with beam polarization, whose main role is that of increasing the production cross-section.

For definiteness we are going to consider two scenarios: an $e^{+} e^{-}$collider inspired by the CLIC design and a futuristic high-energy muon collider. As benchmarks for CLIC we focus on centre-of-mass energies foreseen for the second and third stages of the experiment, namely $1.5 \mathrm{TeV}$ (CLIC-2) and $3 \mathrm{TeV}$ (CLIC-3) [37]. The final luminosities after 27 years of run are estimated to be $2.5 \mathrm{ab}^{-1}$ for CLIC-2 and $5 \mathrm{ab}^{-1}$ for CLIC-3 [38]. One can further benefit from possible electron and positron polarization. The cross-section of a generically polarized $e^{+} e^{-}$beam in terms of the polarization fractions $P_{e^{-}}$and $P_{e^{+}}$is defined by

$$
\begin{aligned}
\sigma_{P_{e^{-}} P_{e^{+}}}= & \frac{1}{4}\left[\left(1+P_{e^{-}}\right)\left(1+P_{e^{+}}\right) \sigma_{R R}+\left(1-P_{e^{-}}\right)\left(1-P_{e^{+}}\right) \sigma_{L L}\right. \\
& \left.+\left(1+P_{e^{-}}\right)\left(1-P_{e^{+}}\right) \sigma_{R L}+\left(1-P_{e^{-}}\right)\left(1+P_{e^{+}}\right) \sigma_{L R}\right],
\end{aligned}
$$

where $\sigma_{L R}$ stands for instance for the cross-section if the $e^{-}$-beam is completely left-handed polarized $\left(P_{e^{-}}=-1\right)$ and the $e^{+}$-beam is completely right-handed polarized $\left(P_{e^{+}}=+1\right)$. For our analysis it turns out to be helpful to have negative $e^{-}$polarization and positive $e^{+}$polarization, since this configuration enhances the $e^{+} e^{-} \rightarrow f \bar{f}$ cross-section. The Higgs program at CLIC prefers $P_{e^{-}}=-80 \%$, while for a measurement of top quark couplings $P_{e^{-}}=+80 \%$ is preferable. It can be assumed that $4 / 5$ of the time will be devoted to the Higgs program so we will use as a benchmark $P_{e^{-}}=-80 \%$, with an effective luminosity of $L=2 \mathrm{ab}^{-1}$ for CLIC-2 and $L=4 \mathrm{ab}^{-1}$ for CLIC-3 [38]. We further rescale the latter luminosities by a 0.6 factor, in order to account for beam effects. ${ }^{7}$ There is also the possibility of positron polarization at a lower level, although positron polarization is not part of the baseline CLIC design [37]. To this end we will consider either $P_{e^{+}}=0 \%$ or $P_{e^{+}}=30 \%$ (the latter corresponding to the configuration employed in table 1).

The option of a muon collider was recently revived ${ }^{8}$ thanks to new proposals for muon sources. In particular, the proposal of a low emittance muon source from positron scattering on a target, LEMMA [21-23], allows to reach centre-of-mass energies above $10 \mathrm{TeV}$ [40]. As a benchmark we choose a $14 \mathrm{TeV}$ collider option with a luminosity of $L=20 \mathrm{ab}^{-1}$. For comparison we vary the luminosity by factors of $1 / 4,1 / 2$ and 4 , and we consider even a higher energy option of $\sqrt{s}=30 \mathrm{TeV}$ for various values of the luminosity. ${ }^{9}$

Following ref. [14] we perform a binned likelihood analysis on the differential cross section of the process $\ell^{+} \ell^{-} \rightarrow f \bar{f}$ with respect to the cosine of the scattering angle $\theta$. In particular, we divide the latter in 10 uniform intervals for $\cos \theta \in[-0.95,0.95]$. For the final states we assume the following detection efficiencies: $100 \%$ for leptons, $80 \%$ for $b$-jets and $50 \%$ for $c$-jets. For our numerical analysis we compute the cross sections at LO. Our bounds are however in good agreement with the ones of ref. [14], which includes NLO corrections. This shows that NLO effects have only a small impact on the results.

\footnotetext{
${ }^{7}$ We thank Jorge De Blas for correspondence about this point.

${ }^{8}$ For a recent phenomenological analysis pointing out the advantages of a muon collider see ref. [39].

${ }^{9}$ We thank Andrea Wulzer for correspondence about possible luminosity benchmarks.
} 

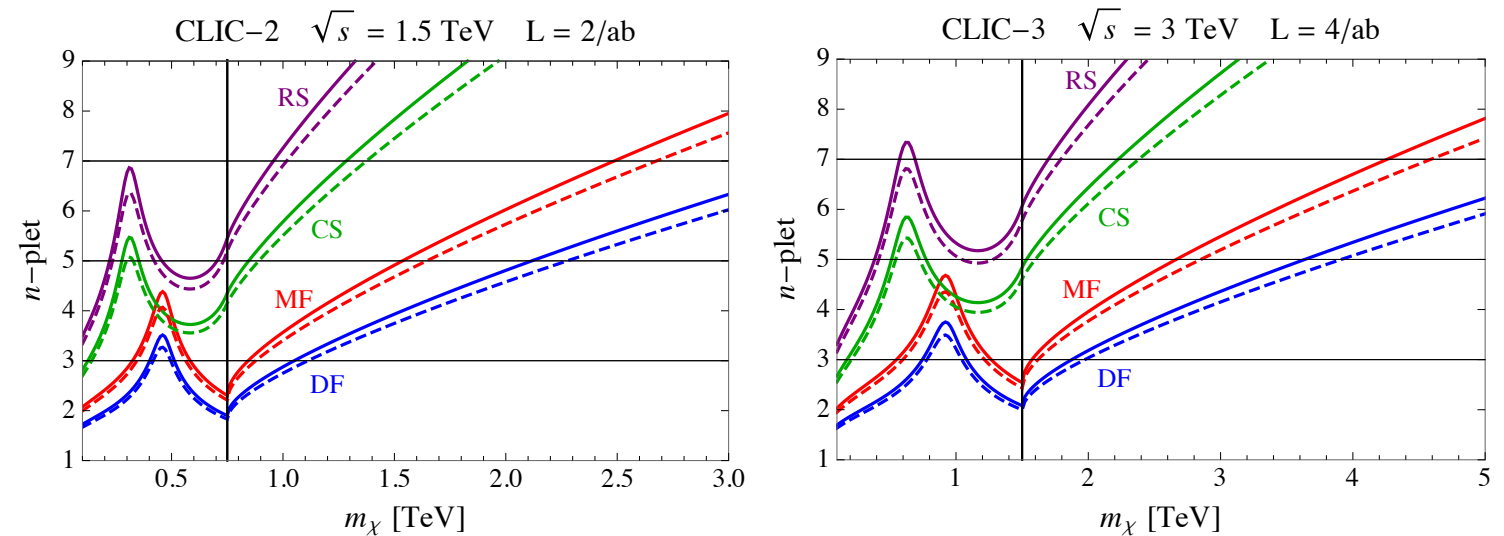

Figure 7. Expected 95\% CL exclusion limits for CLIC-2 (left panel) and CLIC-3 (right panel), for different Lorentz representations and polarization fractions $\left(P_{e^{-}}, P_{e^{+}}\right)=(-80 \%, 0)$ [full lines] and $\left(P_{e^{-}}, P_{e^{+}}\right)=(-80 \%,+30 \%)$ [dashed lines].

\subsection{Results: $e^{+} e^{-}$collider}

The results are displayed in figure 7 where we show the 95\% CL exclusion limits in the plane $\left(m_{\chi}, n\right)$ for different Lorentz representations (RS, CS, MF, DF) and for CLIC-2 $\left(\sqrt{s}=1.5 \mathrm{TeV}, L=2 \mathrm{ab}^{-1}\right)$ and CLIC-3 $\left(\sqrt{s}=3 \mathrm{TeV}, L=4 \mathrm{ab}^{-1}\right)$. To obtain these exclusions we have combined the $e / \mu / b / c$ channels assuming a systematic error of $0.3 \%$, we rescaled the luminosities by a 0.6 factor due to beam effects and considered the polarization fractions $P_{e^{-}}=-80 \%$ and $P_{e^{+}}=0(+30 \%)$.

The vertical black line in both plots denotes the kinematical threshold for pairproduction $\sqrt{s} / 2$. In the region below threshold (on the right side of the vertical black line) the bound on the mass grows with the dimensionality of the multiplet and eventually enters the EFT regime for $m_{\chi} \gg \sqrt{s} / 2$ (cf. figure 1).

The bounds in the region above threshold (on the left side of the vertical black line) have a non-trivial behavior, showing a significant dip in sensitivity. This effect can be traced back to the fact that the real part of the form factor above threshold has an accidental zero (e.g. $x \equiv s / m_{\chi}^{2} \simeq 11$ in the case of the self-energy correction due to fermions). For such value of $s / m_{\chi}^{2}$ the new-physics contributions are strongly suppressed since the interference with the SM vanishes and the corrections only come from the square of the imaginary part of the $\Pi_{S, F}$ form factors. Although a dip in sensitivity arises for certain values of $x$, one can still extract meaningful bounds also above the threshold for pair-production, which can be competitive with direct searches (cf. also section 6). Due to the sensitivity dip, runs at different center of mass energy can be complementary in testing multiplets with relatively low masses. For instance CLIC-3 can not test a $(1,3,0)_{\mathrm{DF}}$ multiplet for masses in the range $[0.8,1.0] \mathrm{TeV}$. This mass range can however be fully tested at CLIC-2.

Regarding the sensitivity to DM candidates, we notice that CLIC-3 (together with CLIC-2) would be able to cover the relevant parameter space of the $(1,3, \epsilon)_{\mathrm{DF}}$ multiplet up to the thermal mass that saturates the DM relic abundance. 


\subsection{Results: muon collider}

For the case of the muon collider we report the results in a slightly different way, emphasizing the role of luminosity. In particular, we report in figures 8-9 the 95\% CL bounds for $\sqrt{s}=14 \mathrm{TeV}$ and $30 \mathrm{TeV}$, and for various Lorentz representations and integrated luminosities (assuming a systematic error of $0.3 \%$ and no polarization).

We notice that a high-energy muon collider can significantly extend the reach with respect to CLIC, provided enough integrated luminosity can be collected. Since the $\ell^{+} \ell^{-} \rightarrow f \bar{f}$ cross section decreases quadratically with the energy, a quadratic increase in the integrated luminosity is needed in order to allow for comparable sensitivity. Smaller luminosities can instead significantly degrade the reach, in particular precluding the possibility to test small multiplets.

Analogously to what we saw for CLIC, the bounds for multiplets with a mass roughly $1 / 4$ of the collider energy suffer from a decreased sensitivity due to a suppression of the interference with the SM amplitude. Collider runs at different energies can therefore provide complementary bounds for the small mass ranges.

Finally we notice that a $14 \mathrm{TeV}$ muon collider could be useful to test several DM candidates, namely the $(1,3, \epsilon)_{\mathrm{DF}},(1,5, \epsilon)_{\mathrm{DF}}$ and $(1,7, \epsilon)_{\mathrm{DF}}$ multiplets, even for mass values that saturate the DM relic abundance. It could also probe a significant fraction of the parameter space of the $(1,5,0)_{\mathrm{MF}}$ multiplet. A $30 \mathrm{TeV}$ muon collider could fully probe most of the DM candidates listed in table 1, provided enough integrated luminosity is collected.

\section{Comparison with direct searches}

Direct searches are particularly challenging if the lightest state within the EW multiplet is neutral, while if the lightest component is charged the current bounds are already quite strong. For instance, charged particles which are stable on the detector scale can be searched for by the longer time of flight through the detector and their anomalous energy loss. Current bounds are, depending on the quantum numbers, of the order of 300-900 GeV [27, 41, 42].

If the lightest state within the EW multiplet is neutral and stable, direct searches become more difficult and the bounds weaken. Such states can be searched for in disappearing track searches or mono-X searches. ${ }^{10}$ While a detailed assessment of direct searches is beyond the scope of this paper, we will shortly comment on the relevance of these searches for the EW multiplets considered in our analysis.

Disappearing tracks. The small mass splitting between the components of the EW multiplet implies a rather long lifetime of the next-to lightest charged component. This offers the possibility to search for them at colliders through disappearing charged tracks. In such searches a long-lived charged particle leaves a track in the innermost layers of the detector but not in the layers with higher radii, since it decays in the meanwhile into the neutral stable component of the EW multiplet and into a very soft pion, which cannot be reconstructed. While at Run-1 tracks needed to fly at least $29.9 \mathrm{~cm}$ in the ATLAS detector

\footnotetext{
${ }^{10}$ In non-minimal scenarios with extra states also soft lepton searches can be competitive [43].
} 

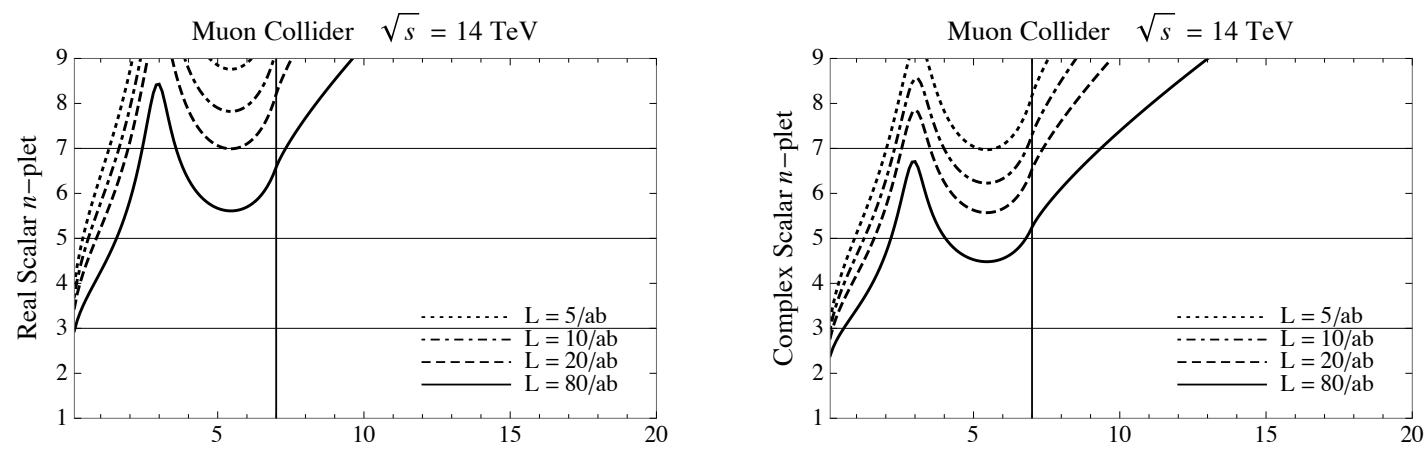

$m_{\chi}[\mathrm{TeV}]$
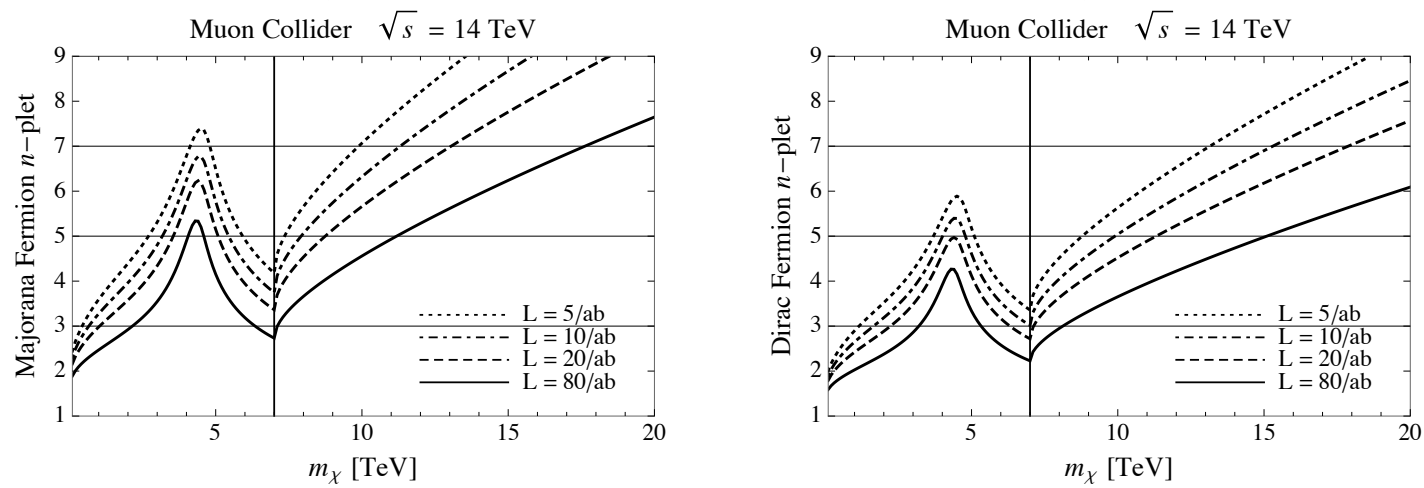

Figure 8. Expected 95\% CL exclusion limits for a $14 \mathrm{TeV}$ muon collider.
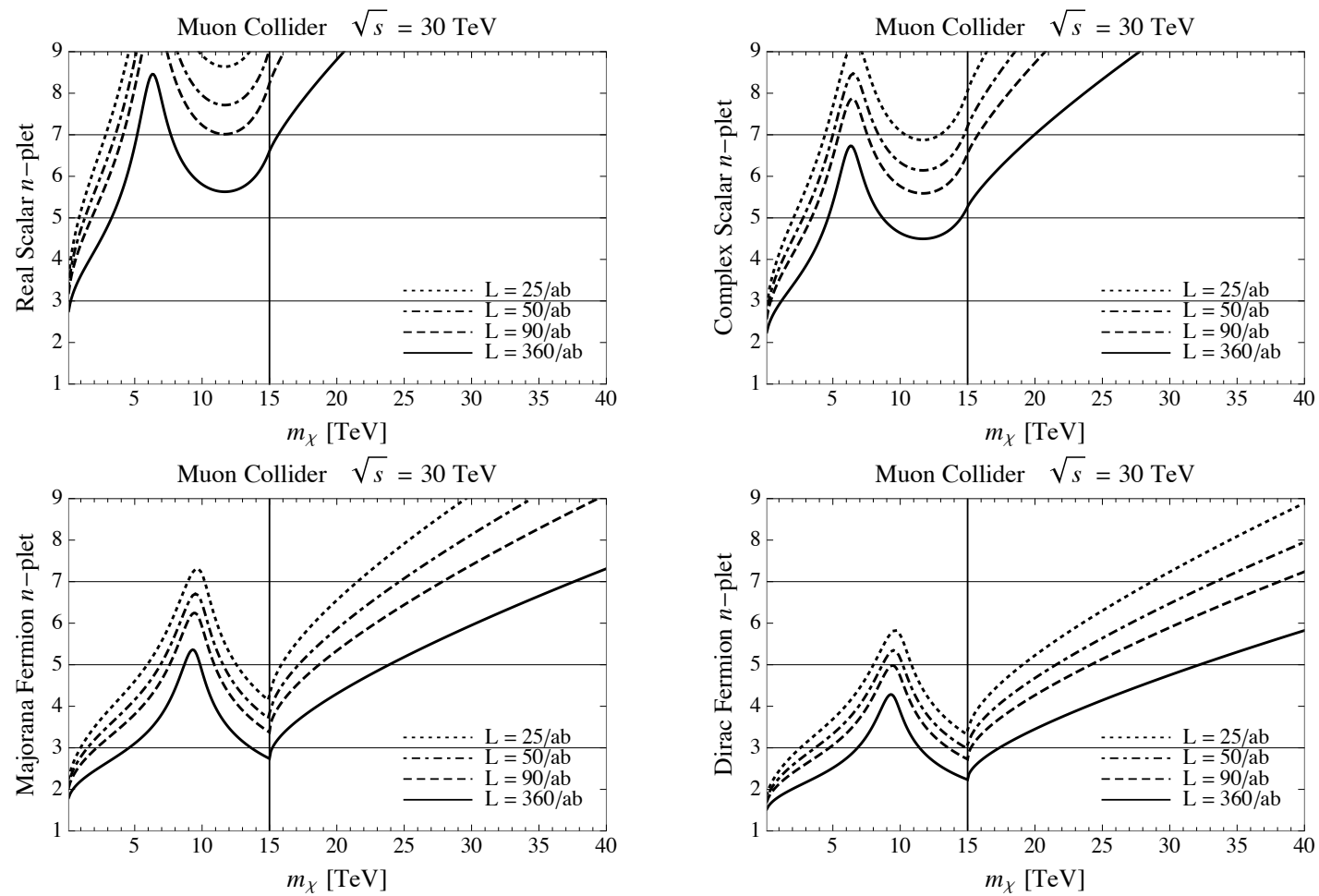

Figure 9. Expected 95\% CL exclusion limits for a $30 \mathrm{TeV}$ muon collider. 
due to the requirement of at least one hit in the $b$-layer and three in the pixel detector [44] (similarly, also for the CMS Run-1 search [45]), the installation of a new innermost layer in the ATLAS detector led to a significant improvement in the region of small lifetimes ( $\sim 0.2 \mathrm{~ns}$ ). For instance, at Run $1 \mathrm{EW}$ triplets ("winos"), have been excluded up to $270 \mathrm{GeV}$ [44], Run-2 could exclude instead masses of $460 \mathrm{GeV}$ [46]. Remarkably, current bounds already outperformed projections on the reach of the HL-LHC before the detector upgrade for the wino [47] and the Majorana 5-plet [48]. For the latter, even though the lifetime is smaller than in the wino case (and hence the track length of the disappearing track is shorter, implying a less efficient search), one can benefit from the higher cross section. The projections derived in ref. [48] for the Majorana 5-plet were in fact stronger than for the wino.

Finally, we mention that in ref. [49] a new search strategy relying only on two hits (instead of four) in the innermost detectors was proposed. The benefit of this approach is a better sensitivity to states with shorter lifetimes (larger multiplets), requiring track lengths of $\gtrsim 5 \mathrm{~cm}$. Following this strategy ref. [49] found that winos can be excluded at the HL-LHC up to $1.2 \mathrm{TeV}$ and EW doublets with $y=1 / 2$ ("higgsinos") up to $550 \mathrm{GeV}$. In ref. [50] it was shown that performing a disappearing track search within the inner 10 $\mathrm{cm}$ of the detector (hence modifying the detector set-up compared to ATLAS and CMS) would significantly improve the reach on higgsinos, allowing the FCC-100 to reach their thermal mass.

Mono-X. Another way of testing EW multiplets in which the lightest component is electrically neutral and stable is by mono-X searches, where $\mathrm{X}$ stands for a high-energy jet, photon or a $W / Z /$ Higgs boson. These searches require events with large missing transverse energy in association with hard SM radiation. While mono-X searches for DM-like states are more model independent, their reach is rather modest. The best sensitivity can usually be obtained from mono-jet searches, which are typically also more sensitive than vector boson fusion processes [47]. Ref. [51] provides a comparison of the reach of disappearing track and mono-jet searches in the case of the wino and higgsino, both at the LHC and at future hadron colliders. It is found that the reach for winos (higgsinos) in monojet searches is $280 \mathrm{GeV}(200 \mathrm{GeV})$ for the HL-LHC, $700 \mathrm{GeV}(490 \mathrm{GeV})$ at a $27 \mathrm{TeV}$ collider and $2 \mathrm{TeV}$ $(1.4 \mathrm{TeV})$ at a $100 \mathrm{TeV}$ collider, while the reach for disappearing track searches is roughly a factor of 3 larger for winos. Instead for higgsinos the disappearing track searches lead only to a slight improvement with respect to the reach of monojet searches. Monojet searches for larger multiplets were studied in ref. [52], where it is found that a MDM quintuplet can be constrained up to masses of roughly $700 \mathrm{GeV}$ at the HL-LHC (with $\sqrt{s}=13 \mathrm{TeV}$ ) and up to $3.8 \mathrm{TeV}$ at a $100 \mathrm{TeV}$ collider with $L=30 \mathrm{ab}^{-1}$.

For $e^{+} e^{-}$machines we note that although direct searches at LEP almost allowed to saturate the kinematical reach for pair production, setting bounds on the mass of electroweakly charged states of the order of $\sqrt{s} / 2-\epsilon$, with $\epsilon \approx 10 \% \sqrt{s}$ [53], this will not necessarily be the case at high-energy lepton colliders like CLIC. The reason is that some SM backgrounds, like the vector-boson-fusion production of a $Z$ boson decaying into neutri- 
nos or the real emission from Bhabha scattering, become more relevant at high energies. ${ }^{11}$ Hence, indirect probes of EW states based on precision measurement become relevant also at energies above the pair-production threshold.

In summary, while direct searches lead to stronger bounds on small multiplets (doublets and triplets), for larger multiplicities (e.g. 5-plets) precision measurements seem to surpass the projected sensitivities from direct searches. A detailed comparison is however beyond the scope of this paper, since a dedicated analysis of direct searches for EW multiplets with $n>3$ after detector upgrades is not available yet in the literature.

\section{Conclusions}

In this paper we investigated the possibility of probing EW multiplets by precision measurements at the LHC and future high-energy hadron and lepton colliders. We first focused on the Drell-Yan production of $\ell^{+} \ell^{-}$and $\ell \nu$ at hadron colliders, which are modified in the presence of new EW multiplets due to one-loop corrections of the gauge boson propagators.

We compared in detail neutral and charged Drell-Yan production and showed that the charged current channel actually leads to much stronger bounds than the previously considered neutral current channel [15]. While we find that an $\mathrm{SU}(2)_{L}$ doublet can neither be probed at the LHC nor at future hadron colliders since the modifications of the SM processes remain below the systematic uncertainty, for larger multiplets the prospects are much more promising. For instance, we find that a future $100 \mathrm{TeV}$ collider can fully probe the DM hypothesis in the case of a $(1,5, \epsilon)_{\mathrm{DF}}$ state. For other EW multiplets the thermal mass cannot be reached but we can still constrain part of the allowed parameter space. The sensitivity on EW multiplets is just below the reach of disappearing track searches, although indirect EW probes are more model-independent (e.g. they do not depend on kinematical features like the lifetime of the inter-multiplet components). A precision measurement of the Drell-Yan process hence provides an alternative, model-independent probe of EW multiplets. By reducing systematic uncertainties, for instance via a better knowledge of PDFs, the reach of our method increases significantly.

Furthermore, we considered the possibility of constraining EW multiplets via the process $\ell^{+} \ell^{-} \rightarrow f \bar{f}$ at a future $e^{+} e^{-}$or $\mu^{+} \mu^{-}$collider. The fundamental difference compared to hadron colliders is that the centre-of-mass energy is fixed. Exclusion bounds thus strongly depend on the shape of the form factors of the new physics contribution to the gauge boson self-energies. Since the sensitivity is not a monotonously decreasing function with the mass of the new multiplet, different centre-of-mass energies can probe complementary mass ranges. Different stages for the centre-of-mass energy are hence potentially helpful for probing new EW particles at lepton colliders. Employing the energy and luminosity benchmarks of CLIC we find that it is possible to reach the thermal mass of a $(1,3, \epsilon)_{\mathrm{DF}}$ multiplet, while for the recently revived option of a high-energy and high-luminosity muon collider also the $(1,5, \epsilon)_{\mathrm{DF}}$ and $(1,7, \epsilon)_{\mathrm{DF}}$ thermal masses can be tested.

\footnotetext{
${ }^{11}$ We thank Roberto Franceschini for clarifications regarding this point.
} 


\section{Acknowledgments}

We are indebted with Roberto Franceschini for triggering this work and for many useful discussions. We also acknowledge helpful discussions with Jorge De Blas, Eugenio Del Nobile, Jonas Lindert, Marco Nardecchia, Paolo Panci, Thomas Rauh, Filippo Sala, Michael Spannowsky and Andrea Wulzer. The work of LDL was supported by the ERC grant NEONAT. RG was partially supported by a COFUND/Durham Junior Research Fellowship under the EU grant number 609412.

Note added. While completing this work a preprint appeared on the arXiv [54] where bounds on EW multiplets for the neutral Drell-Yan channel at a future $100 \mathrm{TeV}$ collider were discussed. As we showed however, the charged current channel leads to stronger bounds than the neutral one also for a future $100 \mathrm{TeV}$ collider.

\section{A Perturbativity}

Large representations $\chi \sim(1, n, y)$ will eventually lead to a breakdown of the perturbative expansion. In principle, to assess the perturbative stability of the bounds extracted in this paper one should consider the two-loop corrections to the EW self-energies [55], which is however beyond our scopes. Nevertheless, we can perform a simple estimate of the domain of perturbativity by requiring that the two-loop correction to the beta function of the gauge coupling $g_{i}$ (either $g$ or $g^{\prime}$ ) does not overcome a certain fraction $f$ of the one-loop contribution. ${ }^{12}$ Focussing on the part of the two-loop correction that dominates either for large $n$ or $y$ (see ref. [57] for complete formulae), we find:

$$
\begin{array}{ll}
\left(\frac{g_{i}}{4 \pi}\right)^{2} 4 C_{2}\left(F_{i}\right) S_{2}\left(F_{i}\right)<f \frac{4}{3} S_{2}\left(F_{i}\right) & \text { (fermions) } \\
\left(\frac{g_{i}}{4 \pi}\right)^{2} 4 C_{2}\left(S_{i}\right) S_{2}\left(S_{i}\right) & <f \frac{1}{3} S_{2}\left(S_{i}\right) \quad \text { (scalars) }
\end{array}
$$

Here, $S_{2}$ and $C_{2}$ denote respectively the Dynkin index and the quadratic Casimir of a given fermionic $(F)$ or scalar $(S)$ representation. For $\mathrm{SU}(2)_{L}$ one has $S_{2}=n\left(n^{2}-1\right) / 12$ and $C_{2}=\left(n^{2}-1\right) / 4$, while for $\mathrm{U}(1)_{y}$ one has $S_{2}=C_{2}=y^{2}$. Hence, in terms of $n$ and $y$ we have

$$
\begin{aligned}
& n<\sqrt{f\left(\frac{4 \pi}{g}\right)^{2} \frac{4}{3}+1} \quad \text { and } \quad y<\sqrt{\frac{f}{3}}\left(\frac{4 \pi}{g^{\prime}}\right) \quad \text { (fermions) } \\
& n<\sqrt{f\left(\frac{4 \pi}{g}\right)^{2} \frac{1}{3}+1} \quad \text { and } \quad y<\sqrt{\frac{f}{12}}\left(\frac{4 \pi}{g^{\prime}}\right) \quad \text { (scalars). }
\end{aligned}
$$

Taking as reference values $g=0.65, g^{\prime}=0.36$ and $f=50 \%$, we find $n<16(8)$ and $y<14(7)$ for fermions (scalars). Note, however, that in the case of Dirac fermions already $n \gtrsim 9$ can develop Landau poles within one order of magnitude from the mass scale of $\chi$ (cf. table 9 in ref. [27]). For this reason we cut our plots below $n=10$.

\footnotetext{
${ }^{12} \mathrm{An}$ alternative criterium is to require $\left|\beta_{g_{i}}^{(1)} / g_{i}\right|<1$ [56], where $\beta_{g_{i}}^{(1)}$ is the one-loop beta function.
} 

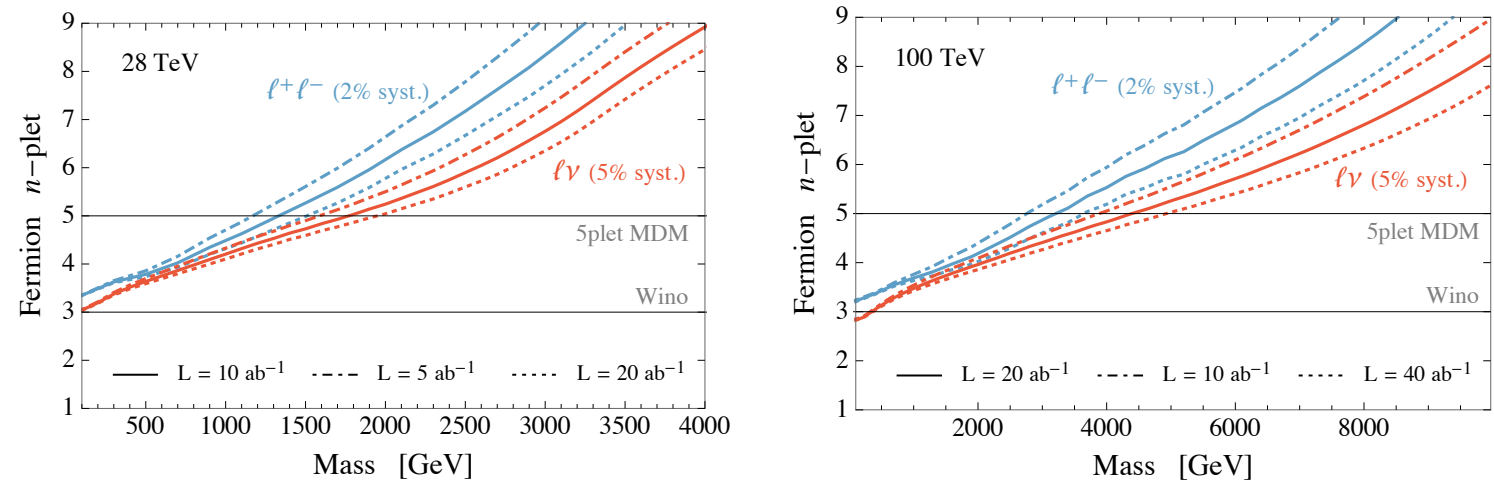

Figure 10. Dependence of the $95 \%$ CL exclusion bounds on the integrated luminosity at the HE-LHC (left panel) and at the FCC-100 (right panel). The red and blue lines correspond to the bounds from the $\ell \nu$ and $\ell^{+} \ell^{-}$final states.
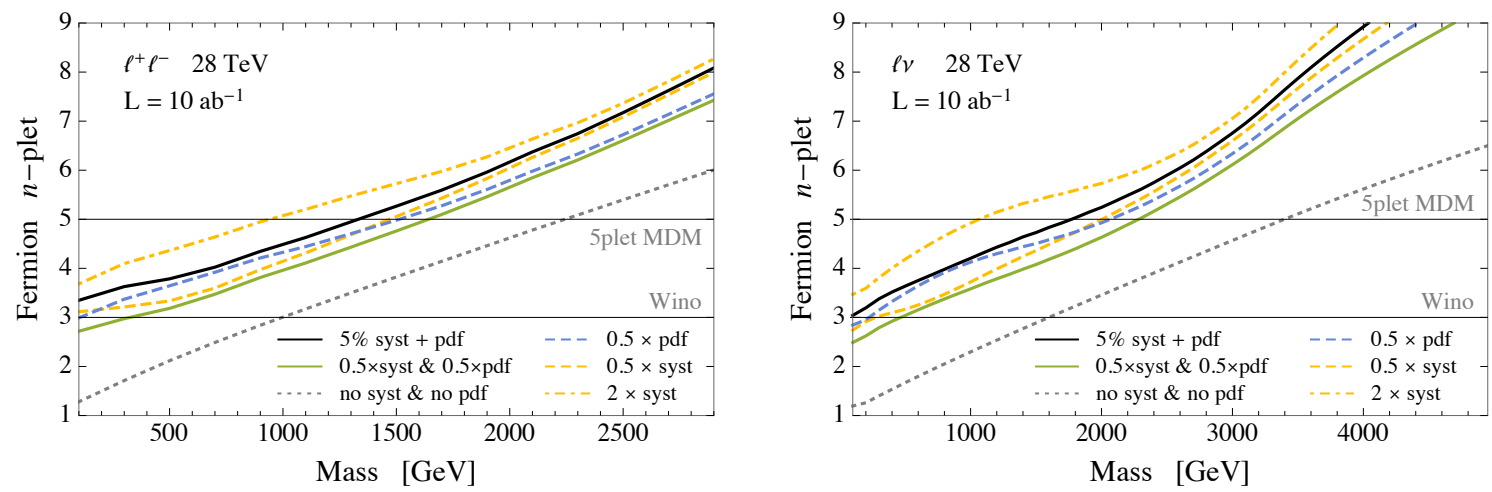

Figure 11. Dependence of the 95\% CL exclusion bounds on Majorana fermion multiplets at the HE-LHC on the PDF uncertainties and on the additional systematic errors.

\section{B Additional results for future hadron colliders}

In this appendix we collect some additional results regarding the expected bounds at highenergy hadron colliders.

In figure 10 we show how the expected bounds for Majorana fermion multiplets depend on the integrated luminosity at the HE-LHC (left panel) and FCC-100 (right panel). The solid lines correspond to the benchmark luminosities used in the main text, while the dotted and dot-dashed lines are obtained by doubling and halving the integrated luminosity respectively. One can see that the impact of a luminosity change is more important for high masses (or equivalently larger multiplets), where the mass bounds change by roughly $\pm 10 \%$ by doubling/halving the luminosity. This behavior is due to the fact that for low masses low luminosities are already enough to give a statistical error below the systematic ones, whereas for larger masses statistical errors still play a relevant role for the benchmark luminosity we considered.

In figures 11 and 12 we show how the expected bounds change by varying our assumptions about PDF and additional systematic errors. The behavior is qualitatively similar to 

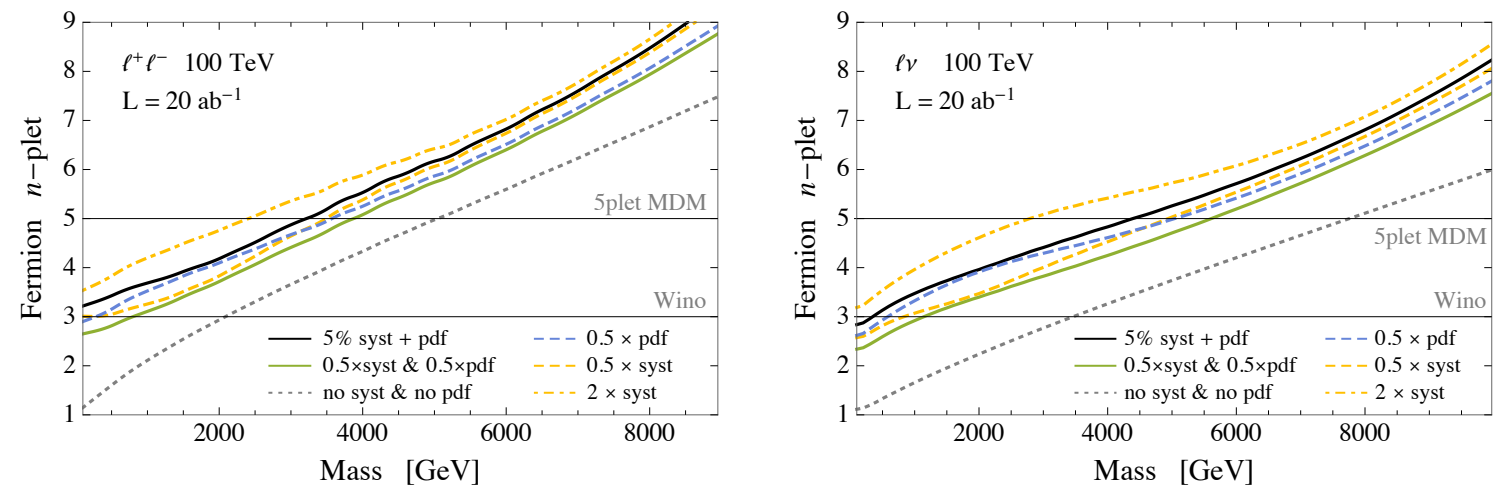

Figure 12. Dependence of the $95 \%$ CL exclusion bounds on Majorana fermion multiplets at the FCC-100 on the PDF uncertainties and on the additional systematic errors.

the one we discussed for the HL-LHC in section 4.2.2. PDF uncertainties tend to dominate for higher masses (above $\sim 2 \mathrm{TeV}$ for HE-LHC and above $\sim 4 \mathrm{TeV}$ for FCC-100), while the additional systematics are more relevant for lower masses. A reduction by $50 \%$ of the systematics allows for a $\sim 15 \%$ improvement in the bounds at high masses and a nearly $100 \%$ improvement for low masses. In particular such an improvement would allow hadron colliders to test Majorana fermion triplets, which can not be probed with the benchmark systematics we assumed in the main text.

Open Access. This article is distributed under the terms of the Creative Commons Attribution License (CC-BY 4.0), which permits any use, distribution and reproduction in any medium, provided the original author(s) and source are credited.

\section{References}

[1] M. Farina, G. Panico, D. Pappadopulo, J.T. Ruderman, R. Torre and A. Wulzer, Energy helps accuracy: electroweak precision tests at hadron colliders, Phys. Lett. B 772 (2017) 210 [arXiv: 1609.08157] [INSPIRE].

[2] V. Cirigliano, M. Gonzalez-Alonso and M.L. Graesser, Non-standard Charged Current Interactions: beta decays versus the LHC, JHEP 02 (2013) 046 [arXiv:1210.4553] [INSPIRE].

[3] J. de Blas, M. Chala and J. Santiago, Global Constraints on Lepton-Quark Contact Interactions, Phys. Rev. D 88 (2013) 095011 [arXiv: 1307.5068] [INSPIRE].

[4] M.E. Peskin and T. Takeuchi, Estimation of oblique electroweak corrections, Phys. Rev. D 46 (1992) 381 [INSPIRE].

[5] R. Barbieri, A. Pomarol, R. Rattazzi and A. Strumia, Electroweak symmetry breaking after LEP-1 and LEP-2, Nucl. Phys. B 703 (2004) 127 [hep-ph/0405040] [InSPIRE].

[6] A. Falkowski, M. González-Alonso and K. Mimouni, Compilation of low-energy constraints on 4-fermion operators in the SMEFT, JHEP 08 (2017) 123 [arXiv:1706. 03783] [INSPIRE].

[7] G. Panico, F. Riva and A. Wulzer, Diboson Interference Resurrection, Phys. Lett. B 776 (2018) 473 [arXiv : 1708.07823] [INSPIRE]. 
[8] R. Franceschini, G. Panico, A. Pomarol, F. Riva and A. Wulzer, Electroweak Precision Tests in High-Energy Diboson Processes, JHEP 02 (2018) 111 [arXiv:1712.01310] [INSPIRE].

[9] S. Banerjee, C. Englert, R.S. Gupta and M. Spannowsky, Probing Electroweak Precision Physics via boosted Higgs-strahlung at the LHC, Phys. Rev. D 98 (2018) 095012 [arXiv: 1807.01796] [INSPIRE].

[10] A. Voigt and S. Westhoff, Virtual signatures of dark sectors in Higgs couplings, JHEP 11 (2017) 009 [arXiv: 1708.01614] [INSPIRE].

[11] D. Becciolini, M. Gillioz, M. Nardecchia, F. Sannino and M. Spannowsky, Constraining new colored matter from the ratio of 3 to 2 jets cross sections at the LHC, Phys. Rev. D 91 (2015) 015010 [Addendum ibid. D 92 (2015) 079905] [arXiv:1403.7411] [InSPIRE].

[12] D.S.M. Alves, J. Galloway, J.T. Ruderman and J.R. Walsh, Running Electroweak Couplings as a Probe of New Physics, JHEP 02 (2015) 007 [arXiv:1410.6810] [INSPIRE].

[13] C. Gross, O. Lebedev and J.M. No, Drell-Yan constraints on new electroweak states: LHC as a $p p \rightarrow l^{+} l^{-}$precision machine, Mod. Phys. Lett. A 32 (2017) 1750094 [arXiv:1602.03877] [INSPIRE].

[14] K. Harigaya, K. Ichikawa, A. Kundu, S. Matsumoto and S. Shirai, Indirect Probe of Electroweak-Interacting Particles at Future Lepton Colliders, JHEP 09 (2015) 105 [arXiv: 1504.03402] [INSPIRE].

[15] S. Matsumoto, S. Shirai and M. Takeuchi, Indirect Probe of Electroweakly Interacting Particles at the High-Luminosity Large Hadron Collider, JHEP 06 (2018) 049 [arXiv: 1711.05449] [INSPIRE].

[16] M. Benedikt and F. Zimmermann, Proton Colliders at the Energy Frontier, Nucl. Instrum. Meth. A 907 (2018) 200 [arXiv:1803.09723] [INSPIRE].

[17] I. Hinchliffe, A. Kotwal, M.L. Mangano, C. Quigg and L.-T. Wang, Luminosity goals for a 100-TeV pp collider, Int. J. Mod. Phys. A 30 (2015) 1544002 [arXiv:1504.06108] [InSPIRE].

[18] T. Golling et al., Physics at a $100 \mathrm{TeV}$ pp collider: beyond the Standard Model phenomena, CERN Yellow Report (2017) 441 [arXiv:1606.00947] [INSPIRE].

[19] L. Linssen, A. Miyamoto, M. Stanitzki and H. Weerts, Physics and Detectors at CLIC: CLIC Conceptual Design Report, arXiv: 1202.5940 [INSPIRE].

[20] J.-P. Delahaye et al., Enabling Intensity and Energy Frontier Science with a Muon Accelerator Facility in the U.S.: A White Paper Submitted to the 2013 U.S. Community Summer Study of the Division of Particles and Fields of the American Physical Society, in Proceedings, 2013 Community Summer Study on the Future of U.S. Particle Physics: Snowmass on the Mississippi (CSS2013), Minneapolis, MN, U.S.A., July 29-August 6, 2013 [arXiv: 1308.0494] [INSPIRE].

[21] M. Antonelli, M. Boscolo, R. Di Nardo and P. Raimondi, Novel proposal for a low emittance muon beam using positron beam on target, Nucl. Instrum. Meth. A 807 (2016) 101 [arXiv: 1509.04454] [INSPIRE].

[22] F. Collamati et al., Low Emittance Muon Beams from Positrons, PoS (NuFact2017)103 (2017).

[23] M. Boscolo et al., Low emittance muon accelerator studies with production from positrons on target, Phys. Rev. Accel. Beams 21 (2018) 061005 [arXiv: 1803.06696] [INSPIRE].

[24] M. Cirelli, N. Fornengo and A. Strumia, Minimal dark matter, Nucl. Phys. B 753 (2006) 178 [hep-ph/0512090] [INSPIRE]. 
[25] M. Cirelli, A. Strumia and M. Tamburini, Cosmology and Astrophysics of Minimal Dark Matter, Nucl. Phys. B 787 (2007) 152 [arXiv:0706.4071] [INSPIRE].

[26] M. Cirelli and A. Strumia, Minimal Dark Matter: Model and results, New J. Phys. 11 (2009) 105005 [arXiv: 0903.3381] [INSPIRE].

[27] L. Di Luzio, R. Gröber, J.F. Kamenik and M. Nardecchia, Accidental matter at the LHC, JHEP 07 (2015) 074 [arXiv: 1504.00359] [INSPIRE].

[28] E. Del Nobile, M. Nardecchia and P. Panci, Millicharge or Decay: A Critical Take on Minimal Dark Matter, JCAP 04 (2016) 048 [arXiv: 1512.05353] [INSPIRE].

[29] A. Mitridate, M. Redi, J. Smirnov and A. Strumia, Cosmological Implications of Dark Matter Bound States, JCAP 05 (2017) 006 [arXiv: 1702.01141] [INSPIRE].

[30] C. Anastasiou, L.J. Dixon, K. Melnikov and F. Petriello, Dilepton rapidity distribution in the Drell-Yan process at NNLO in QCD, Phys. Rev. Lett. 91 (2003) 182002 [hep-ph/0306192] [INSPIRE].

[31] C. Anastasiou, L.J. Dixon, K. Melnikov and F. Petriello, High precision QCD at hadron colliders: Electroweak gauge boson rapidity distributions at NNLO, Phys. Rev. D 69 (2004) 094008 [hep-ph/0312266] [INSPIRE].

[32] K. Melnikov and F. Petriello, The $W$ boson production cross section at the LHC through $O\left(\alpha_{s}^{2}\right)$, Phys. Rev. Lett. 96 (2006) 231803 [hep-ph/0603182] [INSPIRE].

[33] S. Catani, L. Cieri, G. Ferrera, D. de Florian and M. Grazzini, Vector boson production at hadron colliders: a fully exclusive QCD calculation at NNLO, Phys. Rev. Lett. 103 (2009) 082001 [arXiv: 0903.2120] [INSPIRE].

[34] S. Catani, G. Ferrera and M. Grazzini, W Boson Production at Hadron Colliders: The Lepton Charge Asymmetry in NNLO QCD, JHEP 05 (2010) 006 [arXiv:1002.3115] [INSPIRE].

[35] R. Gavin, Y. Li, F. Petriello and S. Quackenbush, FEWZ 2.0: A code for hadronic Z production at next-to-next-to-leading order, Comput. Phys. Commun. 182 (2011) 2388 [arXiv: 1011.3540] [INSPIRE].

[36] NNPDF collaboration, Parton distributions for the LHC Run II, JHEP 04 (2015) 040 [arXiv: 1410.8849] [INSPIRE].

[37] M. Aicheler et al., A Multi-TeV Linear Collider Based on CLIC Technology: CLIC Conceptual Design Report, CERN-2012-007 [inSPIRE].

[38] P. Roloff, CLICdp general meeting, https://indico.cern.ch/event/744124/contributions/3074089/attachments/ 1693854/2725991/clicdp_general_meeting_26_07_2018_roloff.pdf (2018).

[39] D. Buttazzo, D. Redigolo, F. Sala and A. Tesi, Fusing Vectors into Scalars at High Energy Lepton Colliders, JHEP 11 (2018) 144 [arXiv:1807.04743] [INSPIRE].

[40] A. Wulzer, Beyond Standard Model: Where do we go from here?, GGI, Florence, https://indico.cern.ch/event/676835/contributions/3006324/attachments/ 1728574/2792873/wulzer_zanetti-MuonCollider.pdf (2018).

[41] CMS collaboration, Searches for long-lived charged particles in pp collisions at $\sqrt{s}=7$ and $8 \mathrm{TeV}$, JHEP 07 (2013) 122 [arXiv:1305.0491] [INSPIRE].

[42] CMS collaboration, Search for long-lived charged particles in proton-proton collisions at $\sqrt{s}=13 \mathrm{TeV}$, Phys. Rev. D 94 (2016) 112004 [arXiv: 1609.08382] [InSPIRE]. 
[43] A. Bharucha, F. Brümmer and N. Desai, Next-to-minimal dark matter at the LHC, JHEP 11 (2018) 195 [arXiv: 1804.02357] [INSPIRE].

[44] ATLAS collaboration, Search for charginos nearly mass degenerate with the lightest neutralino based on a disappearing-track signature in pp collisions at $\sqrt{s}=8 \mathrm{TeV}$ with the ATLAS detector, Phys. Rev. D 88 (2013) 112006 [arXiv:1310.3675] [INSPIRE].

[45] CMS collaboration, Search for disappearing tracks in proton-proton collisions at $\sqrt{s}=8 \mathrm{TeV}$, JHEP 01 (2015) 096 [arXiv:1411.6006] [INSPIRE].

[46] ATLAS collaboration, Search for long-lived charginos based on a disappearing-track signature in pp collisions at $\sqrt{s}=13$ TeV with the ATLAS detector, JHEP 06 (2018) 022 [arXiv: 1712.02118] [INSPIRE].

[47] M. Cirelli, F. Sala and M. Taoso, Wino-like Minimal Dark Matter and future colliders, JHEP 10 (2014) 033 [Erratum ibid. 01 (2015) 041] [arXiv: 1407.7058] [INSPIRE].

[48] B. Ostdiek, Constraining the minimal dark matter fiveplet with LHC searches, Phys. Rev. D 92 (2015) 055008 [arXiv: 1506.03445] [INSPIRE].

[49] H. Fukuda, N. Nagata, H. Otono and S. Shirai, Higgsino Dark Matter or Not: Role of Disappearing Track Searches at the LHC and Future Colliders, Phys. Lett. B 781 (2018) 306 [arXiv: 1703.09675] [INSPIRE].

[50] R. Mahbubani, P. Schwaller and J. Zurita, Closing the window for compressed Dark Sectors with disappearing charged tracks, JHEP 06 (2017) 119 [Erratum ibid. 10 (2017) 061] [arXiv: 1703.05327] [INSPIRE].

[51] T. Han, S. Mukhopadhyay and X. Wang, Electroweak Dark Matter at Future Hadron Colliders, Phys. Rev. D 98 (2018) 035026 [arXiv:1805.00015] [INSPIRE].

[52] Q.-H. Cao, T. Gong, K.-P. Xie and Z. Zhang, Measuring Relic Abundance of Minimal Dark Matter at Hadron Colliders, arXiv:1810.07658 [INSPIRE].

[53] OPAL collaboration, Search for nearly mass degenerate charginos and neutralinos at LEP, Eur. Phys. J. C 29 (2003) 479 [hep-ex/0210043] [InSPIRE].

[54] S. Chigusa, Y. Ema and T. Moroi, Probing Electroweakly Interacting Massive Particles with Drell-Yan Process at 100 TeV Hadron Colliders, Phys. Lett. B 788 (2019) 494 [arXiv: 1810.07349] [INSPIRE].

[55] A. Djouadi and P. Gambino, Electroweak gauge bosons selfenergies: Complete QCD corrections, Phys. Rev. D 49 (1994) 3499 [Erratum ibid. D 53 (1996) 4111] [hep-ph/9309298] [inSPIRE].

[56] F. Goertz, J.F. Kamenik, A. Katz and M. Nardecchia, Indirect Constraints on the Scalar Di-Photon Resonance at the LHC, JHEP 05 (2016) 187 [arXiv:1512.08500] [INSPIRE].

[57] M.E. Machacek and M.T. Vaughn, Two Loop Renormalization Group Equations in a General Quantum Field Theory. 1. Wave Function Renormalization, Nucl. Phys. B 222 (1983) 83 [INSPIRE]. 